\title{
A Path to EUV Photoresist Reference Metrology Using Restricted Tilt Electron Tomography
}

Andrew Barnum ${ }^{1}$, Mark Biedrzycki ${ }^{1}$ and Alain Moussa ${ }^{2}$

${ }^{1}$ Thermo Fisher Scientific, Hillsboro, Oregon, United States, ${ }^{2}$ imec, Leuven, Vlaams-Brabant, Belgium

With extreme ultraviolet (EUV) lithography entering regular use in the semiconductor industry, new techniques are required to tackle the challenges of process monitoring and critical dimension analysis [1]. Inline automated SEM metrology systems have typically been used to estimate process parameters, supplemented by atomic force microscopy (AFM) and other optical techniques. However, all of these methods suffer drawbacks at the feature length scales encountered with EUV processing. CDSEM becomes limited by the resolution of the system as well as the effects of beam induced specimen degradation. AFM data provides excellent resolution for measurements of pitch and the line surface roughness, but suffers from both relatively long acquisition times and the inability of the scanning tip to reach the bottom of the spaces between resist lines. Previous work has established a reference metrology approach using atomic layer deposition (ALD) prior to preparation of both lamella cross-section and planview specimens using the focused ion beam (FIB) [2, 3]. Unfortunately, this approach suffers from a number of drawbacks, principally due to the difficulty of producing planview specimens for the direct measure of structures in a geometry matching that of inline systems. To counteract these issues, a method for acquiring and analyzing dense line structures using TEM tomography has been developed.

The workflow requires sacrificing a wafer for measurements, meaning the proposed method primarily provides a reference metrology for both CDSEM and AFM. The process begins with in-fab photoresist encapsulation using TiOx ALD to stabilize the photoresist prior to dual-beam lamella preparation. This ALD layer protects the resist structures from damage during the initial imaging and protective layer deposition used in the FIB. Specimen thicknesses were targeted at $250 \mathrm{~nm}$ to capture a large amount line structure before degrading image quality for a TEM with an accelerating voltage of $200 \mathrm{kV}$.

As seen in the figure, both the CDSEM and AFM images are of relatively low resolution compared to images provided in TEM. This fact can be exploited in designing the tilt series experiment: given the low target resolution and the high sensitivity of the specimens to radiation damage, a limited tilt series in the range of $+/-12$ degrees has been found to be generally sufficient for generating a reconstructed volume with sufficient fidelity to provide reference metrology for both inline measurement methods. Limitation of the tilt range and restriction of imaging to bright field TEM enables an acquisition time of under 10 minutes, significantly limiting degradation due to radiolysis and other beam damage artifacts. Several samples can be imaged and reconstructed in an automated pipeline, with representative results shown in the figure. From the limited tilt series, sufficient projection data was collected to enable reconstruction of an orthogonal view to the on-zone axis TEM image, yielding the equivalent views to those seen in the CDSEM and AFM, but with the added benefit of slicing through the volume. The effects of the structure shape and roughness variations can now be separated from the instrumental effects for inline measurements.

Ultimately, carefully designed restricted tilt tomography provides an efficient route to generating reference metrology for inline measurements in the fab, and may be extended to any number of structures where users are willing to exchange resolution for a substantial increase in acquisition speed. 


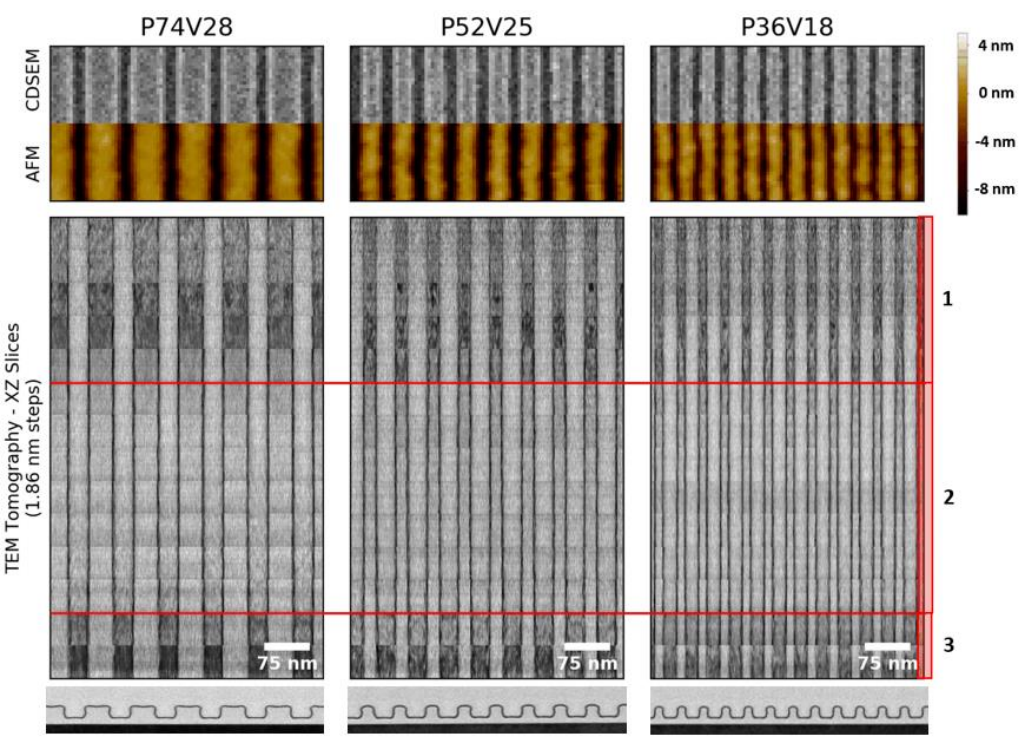

\begin{tabular}{|l|c|c|}
\hline Sample & Pitch $(\mathrm{nm})$ & Linewidth $(\mathrm{nm})$ \\
\hline P74V28 & 74 & 28 \\
\hline P52V25 & 52 & 25 \\
\hline P36V18 & 36 & 18 \\
\hline
\end{tabular}

Figure 1. For three sites on a $300 \mathrm{~mm}$ wafer, initial imaging using inline methods (CDSEM and AFM) were used before preparing lamella of the TiOx ALD stabilized EUV resist structures. Shown below the CDSEM and AFM images are slices through the tomographic volume from the limited tilt series $(+/-12$ degrees) acquired from the lamella. At the bottom of the slice series, the on-zone axis images from each tilt series is shown. The table provides the target dimensions for the resist strctures, and the composition and measurement regions are labeled in the inset TEM bright field image.

\section{References}

[1] Kawada, H., Ebizuka, Y., Sutani, T., and Kawasaki, T., "LER and LWR measurements used for monitoring wiggling and stochastic-failure (Conference Presentation)," in Metrology, Inspection, and Process Control for Microlithography XXXIII, Ukraintsev, V. A. and Adan, O., eds., 10959, International Society for Optics and Photonics, SPIE (2019).

[2] Takamasu, K., Takahashi, S., Kawada, H., and Ikota, M., "Line-width roughness of advanced semiconductor features by using FIB and planar-TEM as reference metrology," in Metrology, Inspection, and Process Control for Microlithography XXXII, Ukraintsev, V. A., ed., 10585, 48 - 55, International Society for Optics and Photonics, SPIE (2018).

[3] Takamasu, K., Takahashi, S., Kawada, H., Ikota, M., Decoster, S., Lazzarino, F., and Lorusso, G., "Linewidth and roughness measurement of SAOP by using FIB and Planer-TEM as reference metrology," in Metrology, Inspection, and Process Control for Microlithography XXXIII, Ukraintsev, V. A. and Adan, O., eds.,10959, 113 - 120, International Society for Optics and Photonics, SPIE (2019). 